Z. Klin. Chem. Klin. Biocbem.

11. Jg. 1973 , S. $291-296$

\title{
Zur Differenzierurg des Lp(a)-Lipoproteins mit Hilfe der Agarosegel-Elektrophorese1)
}

\author{
Von K. H. Vogelberg, G. Utermann und F. A. Gries \\ 2. Medizinische Klinik und Poliklinik der Universität Düsseldorf und Institut für Humangenetik \\ der Universität Marburg
}

(Eingegangen am 4. Dezember 1972/16. März 1973)

Das $L_{p}(a)$-Lipoprotein des menschlichen Serums $(d=1,050-1,090 \mathrm{~g} / \mathrm{ml}) \mathrm{kann}$ in der Agarosegel-Elektrophorese von anderen Lipoproteinen mit prä- $\beta$-Mobilität $\left.(\mathrm{VLDL})^{2}\right)(\mathrm{d}<1,006 \mathrm{~g} / \mathrm{ml})$ abgetrennt werden.

Bei ausreichender Konzentration im Serum ist es als langsamer wandernde Bande einer prä- $\beta$-Doppelbande nachweisbar. Eine solche Doppelbande wurde in $12 \%$ von 758 hyperlipämischen Seren beobachtet. Sie trat bevorzugt in der Rückbildungsphase von Hyperlipoproteinämien auf.

138 Seren von hyperlipämischen Patienten und 71 von normolipämischen Personen wurden mit Hilfe der Ultrazentrifugation untersucht. Das $\mathrm{LP}_{\mathrm{p}}(\mathrm{a})$-Lipoprotein konnte elektrophoretisch in der Fraktion $\mathrm{d}>1,006 \mathrm{~g} / \mathrm{ml}$ in beiden Gruppen gleich häufig nachgewiesen werden $(35 \%)$, jedoch wurde bei Hyperlipoproteinämien mit vermehrten prä- $\beta$-Lipoproteinen ein klarer Zusammenhang zwischen dem Cholesterin/Triglycerid-Quotienten und dem Auftreten des Lp(a)-Lipoproteins gefunden.

Die klinischen Beobachtungen zeigen, daß das $\mathrm{L}_{\mathrm{p}}(\mathrm{a})$-Lipoprotein bettächtlichen individuellen Konzentrationsschwankungen unterworfen sein kann, die offensichtlich vom funktionellen Zustand des Organismus abhängen.

Die Untersuchung des gereinigten Proteinş bestätigt frühere Beobachtungen über den komplexen Aufbau des $L_{p}(a)-L i p o p r o t e i n s$ aus LDI, $\mathrm{L}_{\mathrm{P}}(\mathrm{a})$-Protein und Albumin. Über seine Lipidzusammensetzung wird berichtet.

\section{The differentiation of $L p(a)$-lipoprotein with the aid of agarose gel electrophoresis}

The Ip(a)-lipoprotein of human serum $(\mathrm{d}=1.050-1.090 \mathrm{~g} / \mathrm{ml})$ can be separated from other lipoproteins that have pre- $\beta$-mobility (VLDL) $(\mathrm{d}<1.006 \mathrm{~g} / \mathrm{ml})$ by agarose gel electrophoresis.

When the serum concentration of this protein is sufficiently high it can be detected as a slowly migrating, double pre- $\beta$-band. A double band of this kind was observed in $12 \%$ of 758 hyperlipaemic sera. It appeared chiefly in the regression phase of hyperlipoproteinaemia. 138 Sera from hyperlipaemic patients and 71 from normolipaemic persons were studied in the ultracentrifuge. The Lp(a)-lipoprotein detected by electrophoresis in the fraction $\mathrm{d}>1.006 \mathrm{~g} / \mathrm{ml}$, occurred with the same frequency in both groups (35\%). In hyperlipoproteinaemia with increased pre- $\beta$-lipoproteins, however, there was a clear corrclation between the cholesterol triglyceride quotient and the appearance of the $\mathrm{Lp}(\mathrm{a})$-lipoprotein.

The clinical observations show that the $\mathrm{L}_{\mathrm{p}}$ (a)-lipoprotein can be subject to considerable individual variations, which appear to depend upon the functional state of the organism.

Studies on the purified protein confirmed earlier observations that $\mathrm{Lp}_{\mathrm{p}}(\mathrm{a})$-lipoprotein is a complex of $\mathrm{LDL}, \mathrm{L}_{\mathrm{p}}$ (a)-protein, and albumin. Studies on its lipid composition are reported.

Bei der lipidelektrophoretischen Trennung von prä- $\beta$ Lipoproteinen werden nicht selten Doppelbanden beobachtet, über deren Bedeutung wenig bekannt ist. Die Beobachtung der Subfraktionen wurde zur Beurteilung eines Lipoproteinmusters nicht berücksichtigt.

Wir haben die langsamer wandernde Subfraktion der prä- $\beta$-Lipoproteine elektrophoretisch differenziert und außerdem ihr Vorkommen und ihre klinische Bedeutung untersucht. Zur Charakterisierung wurde die Fraktion mit Hilfe der Ultrazentrifugation isoliert und für immunologische bzw. lipidchemische Analysen gereinigt.

\section{Material und Methoden}

Die Lipidelektrophorese wurde bei 758 Seren von Personen durchgeführt, bei denen eine Hyperlipoproteinämie bekannt oder behandelt wurde. Bei 4 Personen ist die lipidelektrophoretisch langsamer wandernde Bande der prä- $\beta$-Lipoproteine analysiert worden. Es handelte sich um Seren mit besonders großem Gehalt dieser Lipoproteinfraktion, nämlich von
1. einer 53jährigen Patientin (E. G.) mit dekompensierter Herzinsuffizienz (Stauungsleber) bei kombiniertem Mitralvitium (Körpergewicht $8 \%$ unter dem Normgewicht, Triglyceride $1,07 \mathrm{mmol} / 1$, Cholesterin $4,65 \mathrm{mmol} / \mathrm{l}$ )

2. einem 38jährigen Patienten (G. K.) mit Hyperlipoproteinämie bei Verdacht auf Alkoholabusus (normgewichtig, Triglyceride $2,81 \mathrm{mmol} / \mathrm{l}$, Cholesterin $8,00 \mathrm{mmol} / \mathrm{l}$, Therapie $60 \mathrm{mg} \mathrm{DL}-\mathrm{Me}-$ thyl-Thyroxin-Athylester/Tag)

3. einem 62jährigen gesunden Mann (M. A.), der aus Gründen einer altersentsprechenden Arteriosklerose eine polyensäurereiche Diät zu sich nahm (normgewichtig, Triglyceride $1,43 \mathrm{mmol} / \mathrm{l}$, Cholesterin $6,58 \mathrm{mmol} / \mathrm{l}$ )

4. einer 25jährigen Patientin (S. M.) mit einer Anorexia nervosa (19\% unter dem Normgewicht, Triglyceride $1,13 \mathrm{mmol} / \mathrm{l}$, Cholesterin $4,38 \mathrm{mmol} / 1)$.

1) Teilweise vorgetragen beim Symposion über „Struktur, Stoffwechsel und klinische $\Lambda$ spekte der Lipoproteine des Blutes" Ulm, Oktober 1971.

2) Verwendete Abkürzungen:

VLDL: Very low density lipoproteins $(\mathrm{d}<1,006)$;

LDL: Low density lipoproteins $(1,006<d<1,063)$;

HDL: High density lipoproteins $(1,063<\mathrm{d}<1,210)$. 
Lipoprotein-Agarosegel-Elcktrophoresc

Dic Lipidelektrophorese ist mit einer Modifikation der Methode nach RAPP und KAHLKE (1) in albuminhaltigem Agarosegel durchgeführt worden: $2,0 \mathrm{~g}$ Agarose ( $\mathrm{Fa}$. Serva $\mathrm{AG}$, Heidelberg) und $0,25 \mathrm{~g}$ Agar (Behringwerke AG, Marburg) wurden mit $125 \mathrm{ml}$ bidest. Wasser in einem $250 \mathrm{ml}$ Meßkolben durch kurzes Aufkochen (Wasserbad) gelöst und nach Abkühlen auf $52^{\circ} \mathrm{C}$ mit einem $0,1 \mathrm{~mol} / 1$ Veronal-HCl Puffer ( $\mathrm{pH} 8,2$ ) aufgefüllt, in dem $4 \mathrm{~g} / \mathrm{l}$ Rinderalbumin enthalten waren (Behringwerke, s. 'o.). Je $70 \mathrm{ml}$ des Gels wurden auf vorliegende Glasplatten der Elektrophoresekammern gegeben und nach dem Erstarren zur Aufnahme der Serumproben $(20 \mu \mathrm{l})$ ausgestanzt. Die Elektrophorese erfolgte bei 180 Volt und wurde beendet, wenn das einer Serumprobe zugegebene Bromthymolblau eine Trennstrecke von $6,0 \mathrm{~cm}$ erreicht hatte. Nach dem Fixieren in einem $20 \mathrm{~g} / 1$ Essigsäurebad ivurde die Gelschicht auf einer Cellophanfolie getrocknet und angefärbt (2).

\section{Ultrazentrifugation}

Die präparative Ultrazentrifugation wurde mit einer SpincoZentrifuge (Modell L, Rotor 50) innerhalb von $20 \mathrm{~h}$ bei $+4^{\circ} \mathrm{C}$ und $105000 \mathrm{~g}$ durchgeführt; die Einstellung der Serumdichte erfolgte nach HAver et al. (3). Nach der Zentrifugation des Nativserums wurden die flottierenden VLDL mit der Spritze abgezogen, das Sediment zunächst auf eine Dichte von $1,050 \mathrm{~g} / \mathrm{ml}$ eingestellt und das Flotat nach erneuter Zentrifugation ebenfalls abgezogen. Dann wurde die Dichte des verbleibenden Serumrestes auf $1,090 \mathrm{~g} / \mathrm{ml}$ vergrößert und das bei dieser Dichte flottierende Zentrifugat mit einem "tube slicer" (Nuclear Supply and Service Co., Washington D. C.) abgetrennt. Verunreinigende HDI wurden nach wiederholter Zentrifugation bei dieser Dichte beseitigt und das erhaltene Flotat schließlich als farblose Lipoproteinfraktion gegen $9 \mathrm{~g} / 1 \mathrm{NaCl}$-Lösung dialysiert. Die Reinheit der Fraktion wurde lipidelektrophoretisch und immunologisch geprüft. Aufgrund ihrer großen Ausgangskonzentration konnte die Fraktion bei den genannten Personen mit Hilfe der beschriebenen Ultrazentrifugation rein dargestellt werden.

\section{Immunologische Untersuchungen}

Zur immunologischen Charakterisierung wurden die folgenden Antiseren benutzt: Anti- $\beta$-Lipoproteinserum (Immunisierungsmaterial: Serumfraktion $\left(d=1,050 \mathrm{~g} / \mathrm{ml}\right.$ ) eines $L_{p}(a)$-negativen Spenders), Anti-Lp(a)-Serum, Anti-HDL-Serum (sämtlich aus dem Institut für Humangenetik, Marburg/Lahn), Anti- $\alpha_{1}-$ Lipoproteinserum und Anti-Humanalbumin (Behringwerke, s. o.). Die Reinheit der isolierten Fraktion und ihre Identität wurden im Doppeldiffusionstest nach den von UtermanN und WIEgANDT (4) angegebenen Kriterien geprüft. Zur weiteren Sicherung der

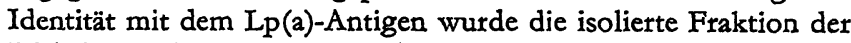
Diskelektrophorese in $37,5 \mathrm{~g} / 1$ Polyacrylamidgelen unterworfen (5). Die vorgefärbten Banden wurden anschließend aus den Gelen ausgeschnitten und nach Einbetten in Agar im Doppeldiffusionstest untersucht. Die immunologische Identität wurde mit isoliertem $L_{P}(a)$-Lipoprotein (6) geprüft.

\section{Lipidanalysen}

\section{Kolorimetrische Bestimmungen}

Der Cholesterin- (7), Glycerid-Glycerin- (8), Phosphot- (9) und Plasmalogengehalt (10) der isolierten Lipoproteine wurde quantitativ erfaßt und die Konzentration im Molverhältnis der einzelnen Lipide berechnet. Für die Berechnung wurde bei den Glyceriden das Molekulargewicht von 885 (8), bei den Plasmalogenen das von 750 (11) zugrunde gelegt.

\section{Chromatographische Untersuchungen}

Die Untersuchungen wurden aus dem nach Folcri et al. (12) extrahierten Lipidgemisch vorgenommen. Dịe in der Unterphase des Extraktes enthaltenen Lipide wurden durch eine PetrolätherGummidialyse (13) in neutrale (Diffusat) und komplexe Lipide (Diffusatrückstand) getrennt und die Lipidfraktionen dünnschichtchromatographisch bzw. nach saurer Methanolyse (14) auch gaschromatographisch analysiert (Abb. 1).
LP(a)-LIPOPROTEIN (LYOPHILISIERT)

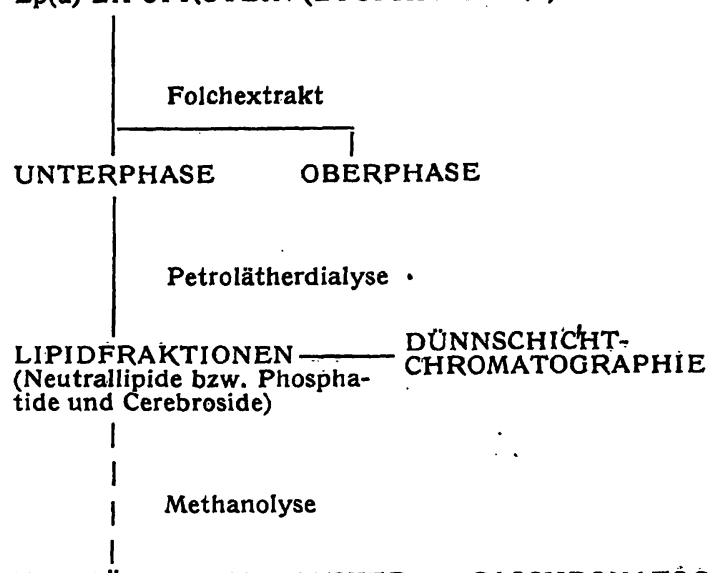

\section{FETTSÄUREMETHYLESTER — GASCHROMATÓGRAPHIE}

Abb. 1

Untersuchungsschema zur Lipidanalyse des Lp(a)-Lipoproteins. Durchgezogene Linie $=$ Trennungsvorgänge, gestrichelte Linie $=$

\section{a) Dünnschichtchromatographie:}

Neutrallipide wurden auf Kieselgel $H$ (Merck AG, Darmstadt) in einem nach FreEMAN und West (15) modifizierten Fließmittelsystem getrennt (Abb. 9a) und nach Veraschung mit $100 \mathrm{~g} / \mathrm{kg}$ Schwefelsäure (16) densitometrisch erfaßt. Phosphatide wurden nach dünnschichtchromatographischer Trennung auf Kieselgel HR (Machery und Nagel, Düren) (17) durch die nach BARLETT modifizierte Phosphorbestimmung (9) quantitativ bestimmt. Glykolipide (Cerebroside) sind lediglich qualitativ untersucht worden (18) (Abb. 9b).

b) Gaschromatographie:

Zur Gaschromatographie wurde ein Instrument der Firma Perkin Elmer verwandt (Modelltyp F 20), das mit einem Ionisationsdetektor ausgestattet ist. Trägergas: $\mathrm{N}_{2}$, Durchflußgeschwindigkeit: $30 \mathrm{ml} / \mathrm{min}$ bei $0,6-1,2 \mathrm{~kg} / \mathrm{cm}^{2}$ (Säule $180 \mathrm{~cm}$ lang, Durchmesser $0,2 \mathrm{~cm}$ ). Säulenfüllung: $10 \%$ EGSS-X auf Diaport $80-100$ mesh. Säulentemperatur: $180^{\circ} \mathrm{C}$.

\section{Ergebnisse}

\section{Vorkommen}

Die Untersuchungen ergaben, daß Doppelbanden der prä- $\beta$-Lipoproteine in etwa $12 \%$ aller von uns durchgeführten Lipidelektrophoresen auftreten, etwa gleich häufig in den Seren von Frauen (11,3\%) und Männern $(12,0 \%)$. Bei den Seren handelte es sich um Hyperlipoproteinämieseren. Auffällig ist, daß Doppelbanden bevorzugt im Rückbildungsstadium einer Hyperlipoproteinämie beobachtet werden, vor allem wenn diese durch Äthanol induzierbar (Abb. 2) oder durch polyensäurereiche Reduktionskost bzw. medikamentöse Therapie (z. B. Clofibrat, DL-Methyl-Thyroxin-Äthylester) beeinflußt worden ist (Abb. 3).

$\mathrm{Da}$ die schneller wandernde prä- $\beta$-Bande rasch dissoziiert und ihre Trennstrecke dann scheinbar mit der der langsamer wandernden übereinstimmen kann (Abb. 4), sind Doppelbanden häufig nur bei der Elektrophorese von Frischserum sichtbar; letztere ist deshalb in jedem Fall anzustreben. Bei der Untersuchung alter Seren oder bei schlechter Elektrophoresetechnik kann namentlich die langsamer wandernde Bande als Verdichtung innerhalb einer breiten prä- $\beta$-Bande übersehen werden. 


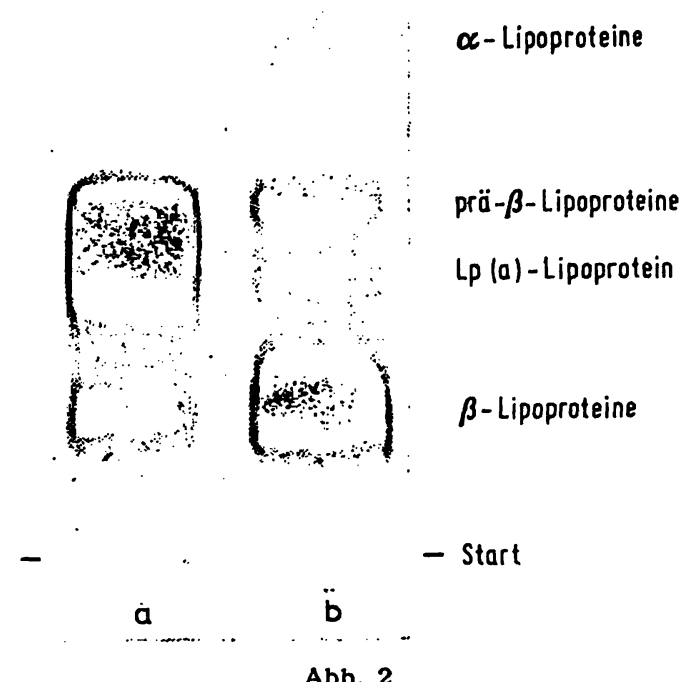

Abb. 2

Veränderungen des Lipoproteinmusters in der Rückbildung einer alkoholinduzierten Hyperlipoproteinämie

a Triglyceride: $6,48 \mathrm{~g} / \mathrm{l} \quad$ Cholesterin: $3,36 \mathrm{~g} / \mathrm{l}$

b Triglyceride: $2,12 \mathrm{~g} / \mathrm{l}$ Cholesterin: $3,50 \mathrm{~g} / \mathrm{l}$

(34jähriger Patient, normgewichtig mit subklinischem Diabetes mellitus. Alkoholkonsum etwa $180 \mathrm{~g} / \mathrm{d}$ )

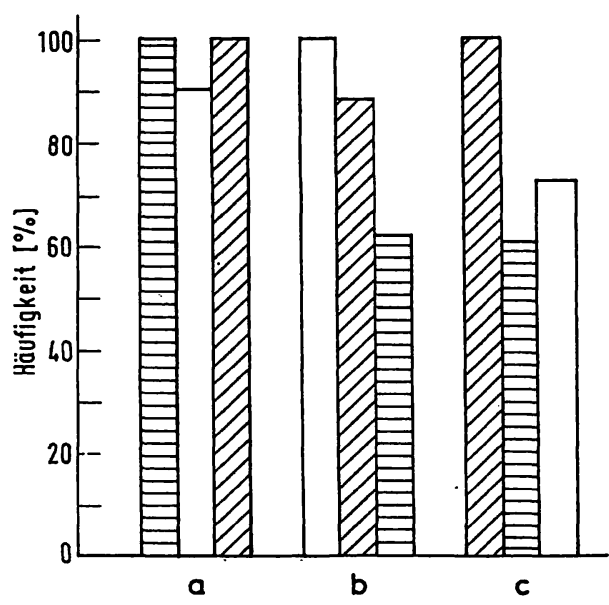

Abb. 3

Die Häufigkeit von Gewichtsabnahme ( $a, n=28)$, Alkoholkarenz $(\mathrm{b}, \mathrm{n}=35$ ) und Hyperlipämiebehandlung ( $\mathrm{c}, \mathrm{n}=49$ ) bei 57 Hyperlipämiepatienten mit prä- $\beta$-Doppelbanden in der Agarosegel-Elektrophorese. Die Differenzierung innerhalb von $a, b$ und $c$ gibt an, wie oft bei einem dieser Merkmale gleichzeitig auch die übrigen vorkommen: waagerecht schraffiert entspricht $a$, weiß b und schräg schraffiert c

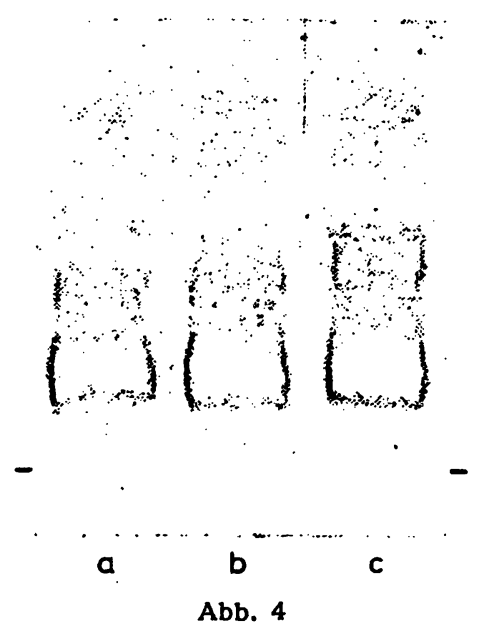

Prä- $\beta$-VLDL einer prä- $\beta$-Doppelbande (a) 10 min nach $i . v$. Injektion von $10 \mathrm{E} / \mathrm{kg}$ Körpergewicht Heparin (b) łbzw. nach Aufbewahren des Nativserums iber $24 \mathrm{~h}$ bei $+4^{\circ} \mathrm{C}$ (c). Triglyceride im Serum $1,56 \mathrm{~g} / \mathrm{l}$, Cholesterin $3,45 \mathrm{~g} / 1$

\section{Charakterisierung}

Die Untersuchung mit der Ultrazentrifuge stellt heraus, daß Lipoproteine der langsamer wandernden Bande eine größere Dichte haben als VLDL. Im Gegensatz zu der schneller wandernden prä- $\beta$-Bande, die bei der Ultrazentrifugation des Nativserums nach $20 \mathrm{~h}$ bei $105000 \mathrm{~g}$ als VLDL flottieren, findet sich diese Fraktion im Unterstand. Die von uns ermittelte Dichte des isolierten Lipoproteins liegt zwischen 1,050 und $1,090 \mathrm{~g} / \mathrm{ml}$. HDL- und LDL-Anteile der Lipoproteinfraktion verhalten sich elektrophoretisch gleich (Abb. 5). Ihre Wanderungsgeschwindigkeit entspricht der im Nativserum sichtbaren, langsamer wandernden Bande der prä- $\beta$-Lipoproteinfraktion.

Bestehen Zweifel über die Identität einer einzelnen prä- $\beta$-Bande - das gilt besonders für Untersuchungen älterer Seren - ist eine genauere Differenzierung durch die Ultrazentrifuge unerläßlich. Bei wiederholter Blutentnahme kann auch ein Heparintest angewandt werden. Abbildung 4 zeigt, daß durch i. v. Injektion von Heparin (10 E/kg Körpergewicht) lediglich die Konzentration der schneller wandernden, $d$. h. VLDLhaltigen prä- $\beta$-Bande vermindert wird; die langsamer wandernde Bande wird durch Heparin elektrophoretisch nicht sichtbar verändert.

Immunologisch ergibt sich, da $\mathrm{B}$ diese Fraktion mit dem von BERG (20) beschriebenen Lp(a)-Antigen identisch ist. Die isolierte Fraktion reagiert im OuchtERLONY-Test sowohl mit Anti-Lp(a)-Serum als auch mit Anti- $\beta$-Lipoproteinserum, wobei beide Präzipitationsbögen bei vergleichender Untersuchung eine Reaktion immunologischer Identität zeigen (4). Die Wanderungsgeschwindigkeit in der Polyacrylamidgel-Elektrophorese entspricht der des $\mathrm{L}_{\mathrm{p}}(\mathrm{a})$-Lipoproteins. Die aus den Polyacrylamidgelen ausgeschnittene vorgefärbte Lipoproteinbande reagiert immunologisch spezifisch mit Anti-Lp(a)- und Anti- $\beta$-Lipoproteinserum (Abb. 7). Einen weiteren Hinweis für die Identität beider Lipoproteine ist das gleichartige Verhalten bei der Lagerung. Wie das $\mathrm{Lp}_{\mathrm{p}}(\mathrm{a})$-Lipoprotein $(21,22)$, dissoziiert das

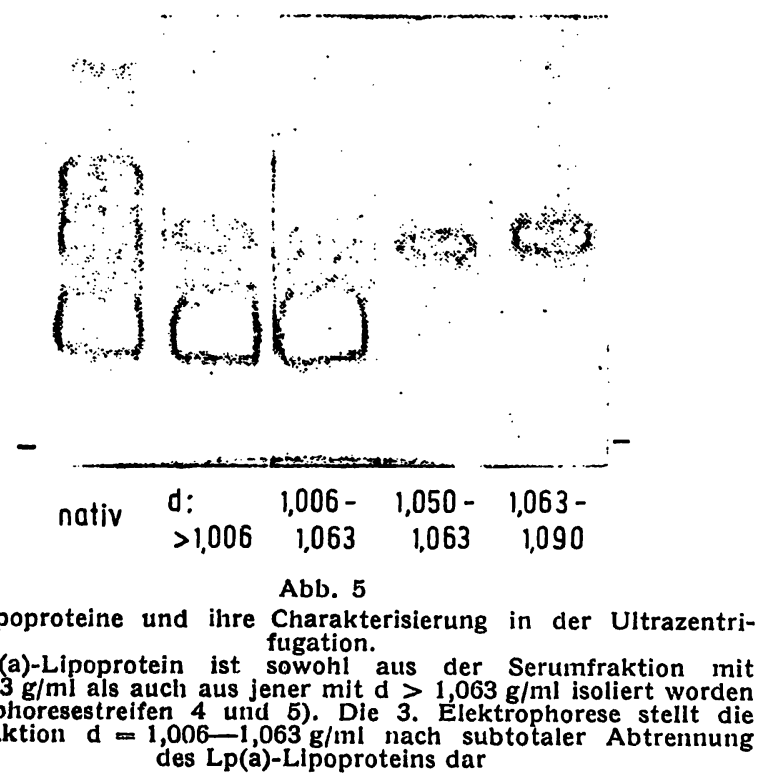




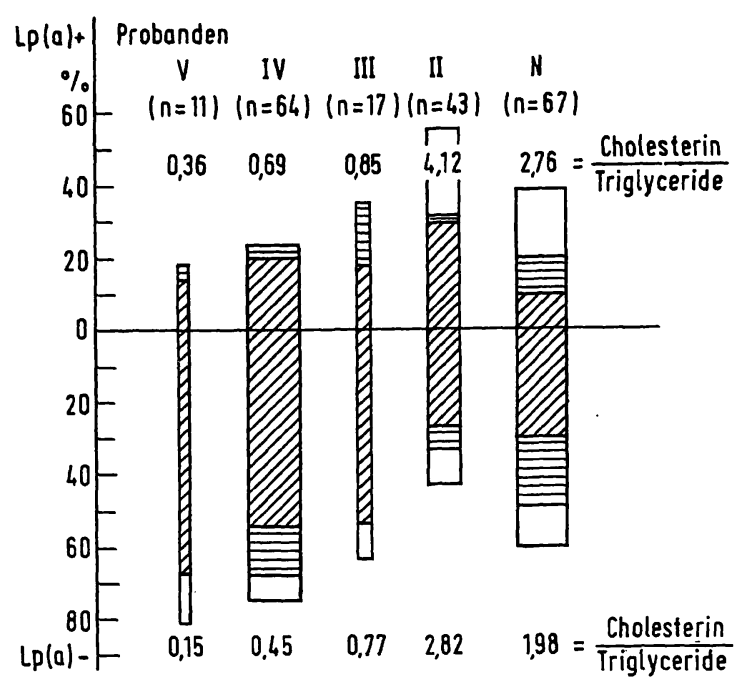

Abb. 6

Häufigkeit des Vorkommens des Lp(a)-Lipoproteins bei 67 Normalpersonen (N) und 138 Hyperlipämie-Patienten des Typs V, IV, III und II mit unterschiedlichem Körpergewicht, gemessen am Lp(a)Nachweis in der Agarosegel-Elektrophorese. (Von den 43 untersuchten eine Hen mit einer Hyperlipoprotenamie des Typs Typ I Hyperlipoeine Hypertriglyceridämie.) Etre

Anteil der untergewichtigen kein L normgewichtigen Anteil der untergewichtigen üchtigen Personen

Die Breite der Säulen entspricht der Anzahl der untersuchten Patienten

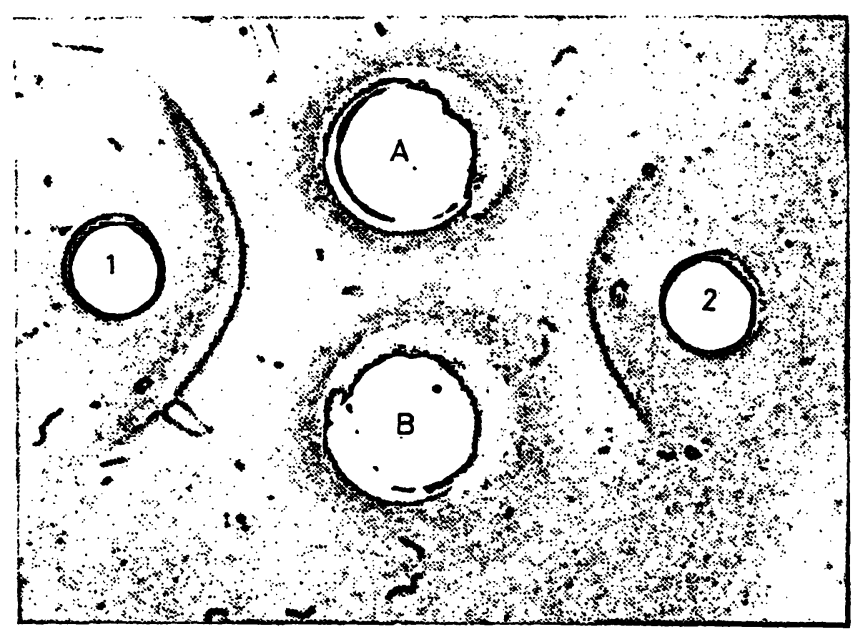

Abb. 7

Immunologisches Verhalten der langsamer wandernden prä- $\beta$-Lipoproteinfraktion und des $L p(a)$-Lipoproteins in Doppeldiffusionswert
(29). A $=$ Lipoproteinfraktion (s. Text), $B=L p(a)$ als Antigen, $1=\mathrm{LDL}$-Antiserum, 2 = Lp(a)-Lipoprotein-Antiserum

langsamer wandernde prä- $\beta$-Lipoprotein während des Aufbewahrens bei $+4^{\circ} \mathrm{C}$ in eine Lipoproteinkomponente mit Eigenschaften der LDL, einen Proteinanteil mit Lp(a)-Antigenität und ein Protein mit immunologischen und elektrophoretischen Eigenschaften von Albumin.

Der quantitativ ermittelte Gehalt des Ip(a)-Lipoproteins an Cholesterin, Glycerid-Glycerin, Phosphor und Plasmal ergibt ein molares Verhältnis von 89,7: 14,3:57,9:1,0. Das freie Glycerin ist nicht bestimmt worden.

Die dünnschichtchromatographisch ermittelte Zusammensetzung von Neutrallipiden und Phosphatiden der Fraktionen verschiedener Personen ist ähnlich. Der Neutrallipid-Gehalt entfällt zu über $60 \%$ auf Cholesterin, $2 / 3$ auf verestertes und $1 / 3$ auf freies Cholesterin. Der Anteil der Glyceride ist gering (Abb. 9a). In der
Tab. 1

Zusammensetzung der Glycerinphosphatide von Lp(a)-Lipoproteinen im Vergleich zu LOW DENSITY Lipoproteinen (LDL) ${ }^{x}$ ). Es wurden pro Analyse $300-400 \mu \mathrm{g}$ des Diffusatrückstandes untersucht (17). Angegeben sind die Minimalwerte und Extremwerte in \%

\begin{tabular}{|c|c|c|}
\hline & $L p(a)$ & LDL') \\
\hline Sphingomyelin & $\begin{array}{c}29,0 \\
(25,7-32,3)\end{array}$ & $\begin{array}{c}25,9 \\
(21,3-29,1)\end{array}$ \\
\hline Lecithin & $\begin{array}{c}53,9 \\
(50,2-57,6)\end{array}$ & $\begin{array}{c}63,7 \\
(54,6-73,7)\end{array}$ \\
\hline Lysolecithin & $\begin{array}{c}10,4 \\
(4,5-16,3)\end{array}$ & $\begin{array}{c}2,7 \\
(1,8-3,8)\end{array}$ \\
\hline Monophosphatinosit & $\begin{array}{c}1,8 \\
(1,2-2,4)\end{array}$ & $\begin{array}{c}1,6 \\
(1,1-2,8)\end{array}$ \\
\hline Serinkephalin & $\begin{array}{c}1,7 \\
(1,0-2,4)\end{array}$ & $\begin{array}{c}0,8 \\
(0,2-1,4)\end{array}$ \\
\hline Colaminkephalin & $\begin{array}{c}1,0 \\
(0,4-1,6)\end{array}$ & $\begin{array}{c}2,2 \\
(1,1-3,9)\end{array}$ \\
\hline $\begin{array}{l}\text { Cardiolipin } \\
\text { (Polyglycerophosphatide) }\end{array}$ & $\begin{array}{c}1,8 \\
(0,2-3,3)\end{array}$ & $\begin{array}{c}2,0 \\
(1,2-3,5)\end{array}$ \\
\hline
\end{tabular}

2) Nach SKIPSKI et al. (39)

Tab. 2

Gaschromatographisch ermittelte Fettsäureverteilung in Neutrallipiden und komplexen Lipiden (Phosphatiden) von $L p(a)$-Lipoproteinen der 4 untersuchten Seren. Der Anteil der Fettsäuren mit ungerader Anzahl von C-Atomen ist in der Tabelle nicht aufgeführt. Er liegt bei beiden Lipidfraktionen zwischen $1-2 \%$

\begin{tabular}{|c|c|c|}
\hline $\begin{array}{l}\text { Anzahl der } \\
\text { C-Atome }\end{array}$ & $\begin{array}{c}\text { Neutrallipide } \\
\%\end{array}$ & $\begin{array}{c}\text { Komplexe Lipide } \\
\%\end{array}$ \\
\hline$<16$ & $4,12 \pm 0,32$ & $4,46 \pm 1,12$ \\
\hline davon: Monoene & $0,76 \pm 0,05$ & $1,49 \pm 0,73$ \\
\hline 16 & $24,97 \pm 2,41$ & $35,50 \pm 3,04$ \\
\hline davon: Monoene & $4,90 \pm 0,35$ & $3,34 \pm 0,41$ \\
\hline 18 & $56,40 \pm 5,70$ & $40,68 \pm 2,53$ \\
\hline davon: Monoene & $25,07 \pm 2,46$ & $13,85 \pm 2,16$ \\
\hline Polyene & $23,72 \pm 2,15$ & $13,91 \pm 085$ \\
\hline$>18$ & $12,77 \pm 1,36$ & $18,13 \pm 1,94$ \\
\hline davon: Monoene & $2,58 \pm 0,61$ & $4,14 \pm 0,52$ \\
\hline Polyene & $2,72 \pm 0,58$ & $3,71 \pm 0,16$ \\
\hline
\end{tabular}

Lp(a)-Fraktion einer Person (E. G.) waren fast ausschließlich Partialglyceride nachweisbar. Die komplexen Lipide enthielten hauptsächlich Lecithin, Sphingomyelin, Lysolecithin und Cerebroside (Abb. 9b). Monophosphatidinosit, Colamin- und Serinkephalin waren nur in Spuren vothanden (Tab. 1).

Die gaschromatographisch ermittelte Fettsäureverteilung ist in Tabelle 2 zusammengestellt. Sie zeigt, daß Neutrallipide sich zu $50-60 \%$ aus Fettsäuren mit $18 \mathrm{C}$-Atomen aufbauen und daß ein beachtlicher Teil (24\%) Polyensäuren sind. In den komplexen Lipiden des Diffusatrückstandes finden sich etwa gleich viel Fettsäuren mit 16 und $18 \mathrm{C}$-Atomen $(30-40 \%)$; der absolute Gehalt an Polyensäuren ist geringer als in den Neutrallipiden.

\section{Beziehungen zum Fettstoffwechsel}

Im Gegensatz zu einer prä- $\beta$-Doppelbande des Nativserums kann das $\mathrm{Lp}$ (a)-Lipoprotein in der Fraktion d $>1,006 \mathrm{~g} / \mathrm{ml}$ elektrophoretisch wesentlich häufiger nachgewiesen werden. Nach der Ultrazentrifugation von 138 Hyperlipämieseren $(20 \mathrm{~h}$ bei $105000 \mathrm{~g})$ wurde sie $47 \mathrm{mal}=34,2 \%$, nach entsprechender Untersuchung 


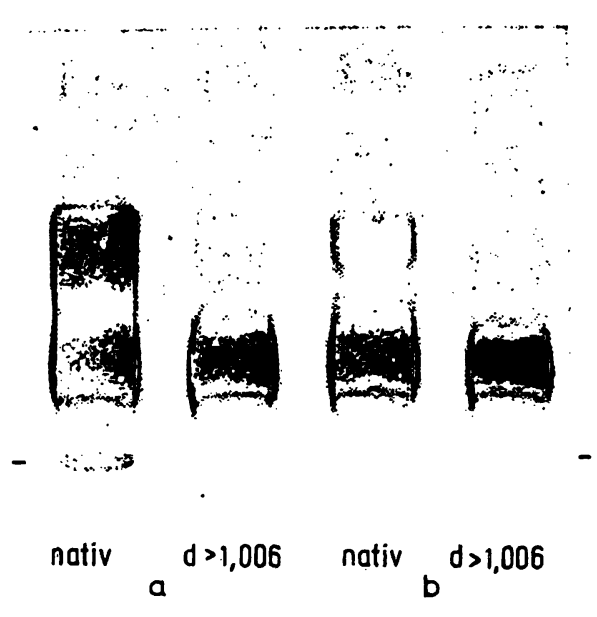

Abb. 8

Individuelle Konzentrationsschwankungen des $L p(a)$-Lipoproteins be einem Hyperlipämie-Patienten (Typ IV) vor (a) und unter lipidsenkender Behandlung mit Clofibrat (b)

Lipide bei a): Triglyceride $5,85 \mathrm{~g} / 1$, Cholesterin $3,50 \mathrm{~g} / \mathrm{l}$ Lipide bei b): Triglyceride $2,49 \mathrm{~g} / \mathrm{l}$, Cholesterin $3,80 \mathrm{~g} /$

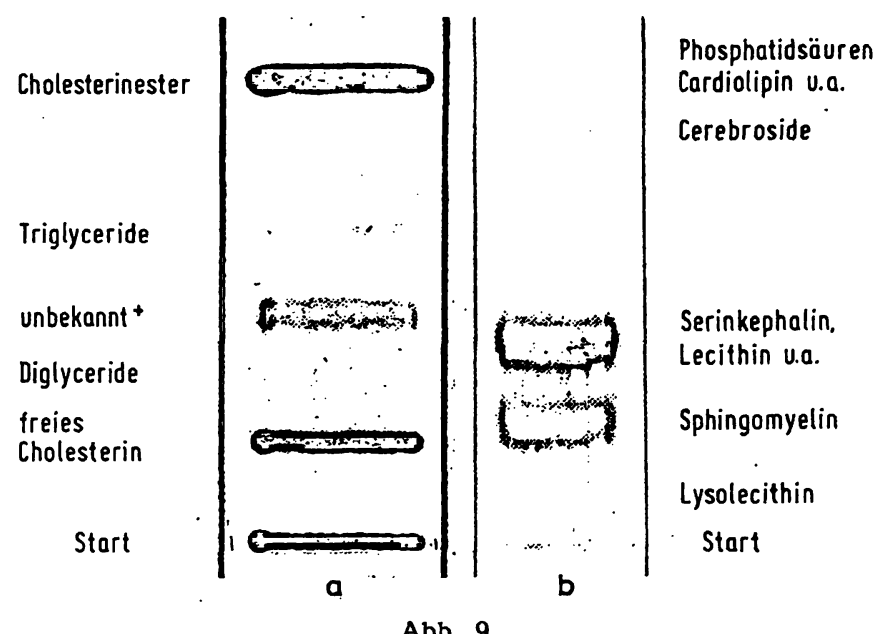

Dünnschichtchromatogramme der $L_{p}(a)$-Lipide von einem der untersuchten Patienten (G. K.)

Die Fraktionen sind unter Behandlung mit DL-Methyl-ThyroxinÄthylester auch in anderen Lipoproteinen nachweisbar

a) Neutrallipide (Diffusat)

1. Fließmittel (15): Benzol/Diäthyläther/Äthanol/Eisessig (Volumina $1501+2001+101+11$ ),

2. Fließmittel: Hexan/Diäthyläther (Volumina $171+31$ )

Fileßfront: $20 \mathrm{~cm}$. Kieselgel H Fertigplatten (Merck, Kat.-Nr. 5715/0025). Im 1. Fließmittel einmal, im 2. zweimal entwickelt. Anfärbung $s$. Text

Aufgetragen etwa $200 \mathrm{mg}$

b) Komplexe Lipide (Diffusatrückstand)

Fließmittel (17): Chloroform/Methanol/Wasser (Volumina $501+211+31) .0,25 \mathrm{~mm}$ Kieselgel $G$ Schicht, Anfärbung in Joddämpfen.

Aufgetragen etwa $320 \mu \mathrm{g}$

von 67 Normalseren (d. h. normolipämischen Seren von gesunden Personen) 25mal beobachtet $(=35,7 \%$, Abb. 6). In 80,7\% aller pathologischen Lipoproteinmuster handelte es sich um Hyperlipämien vom Typ II und IV. Von ihnen wiesen $53,5 \%$ bzw. $23,4 \%$ das Lp(a)-Lipoprotein auf. Bei 10. Patienten des Typs III fand sich das Lipoprotein 6mal, bei 13 des Typs V $2 \mathrm{mal}$. Während bei Adipösen ohne Hyperlipämie höchstens jeder sechste dieses Lipoprotein aufwies, konnte es bei jenen mit Hyperlipämie in positiver Korrelation zum Cholesterin/Triglycerid-Quotienten häufiger beobachtet werden, von Typ II Patienten bei mindestens jedem zweiten.
Die Konzentration des Lp(a)-Lipoproteins ist im Vergleich zu anderen Lipoproteinen gering; trotzdem sind, abgesehen von Unterschieden zwischen verschiedenen Personen, auch individuelle Schwankungen dieser Konzentration festzustellen. Lp(a)-Lipoproteine können beispielsweise bei einem Hyperlipämie-Patienten erst elektrophoretisch nachweisbar sein, nachdem sich dessen Serumtriglyceride deutlich vermindert haben (Abb. 8). Bemerkenswert ist, daß ihr Nachweis im Verlauf einer Normalisierung dieser Fettstoffwechselstörung mit der Zunahme von $\beta$-Lipoproteinen verknüpft ist, während gleichzeitig prä- $\beta$-VLDL verringert werden.

\section{Diskussion}

Über die Existenz einer doppelten bzw. elektrophoretisch mehrfach unterteilten prä- $\beta$-Lipoproteinbande ist bereits wiederholt berichtet worden $(23,24,25)$. Sie wird bevorzugt in der Agarosegel-Elektrophorese beobachtet. Auf Acetatfolien, vor allem auch Papierstreifen, ist sie weniger gut abzugrenzen. Zur Charakterisierung liegen verschiedene Einzelbeobachtungen vor: ElLEFSON et al. (23) konnten zeigen, daß die langsamer wandernden prä- $\beta_{0}$-Lipoproteine eine höhere Dichte besitzen als VLDL. Unabhängig von diesen Befunden wurde von anderen Untersuchern mit dem Lp(a)-Lipoprotein ein Serumlipoprotein isoliert (4, $21,26)$, das nicht den VLDL angehört, aber prä- $\beta$ Mobilität in der Agarosegel-Elektrophorese besitzt (26) und aufgrund seiner spezifischen Dichte auch als „sinking“ prä- $\beta$-Lipoprotein bezeichnet worden ist (25). $\mathrm{Ob}$ es sich um gleiche oder unterschiedliche Lipoproteine handelte, war bisher unklar.

Die vorliegende Untersuchung bestätigt die Befunde von RIDER et al. (27), daß es sich bei dem sog. "sinking“ prä- $\beta$-Lipoprotein um ein $L_{\mathrm{p}}(\mathrm{a})$-Lipoprotein handelt. Sie zeigt darüber hinaus, daß das $\mathrm{L}_{\mathrm{p}}(\mathrm{a})$-Lipoprotein der elektrophoretisch langsamer wandernden prä- $\beta$-Bande identisch ist. Diese kann bei routinemäßiger elektrophoretischer Untersuchung des Serums eindeutig und reproduzierbar von den prä- $\beta$-Lipoproteinen sehr geringer Dichte (VLDL) differenziert werden.

Die Häufigkeit der prä- $\beta$-Doppelbande im Serum entspricht mit $12 \%$ der von RIDER et al. (27) berichteten Frequenz des „sinking“ prä- $\beta$-Lipoproteins (11\%). Unsere zusätzliche Differenzierung durch die Ultrazentrifuge zeigt, daß „sinking“ prä- $\beta$-Lipoproteine elektrophoretisch sogar bei über $34 \%$ aller Seren nachweisbar sind. Diese Frequenz entspricht etwa der immunologisch bestimmten Häufigkeit des $\mathrm{Lp}(\mathrm{a})$ Antigens durch BERG (28). Das antigenwirksame Lipoprotein ist zwar in hyper- und normolipämischen Seren etwa gleich häufig zu beobachten (27), innerhalb der einzelnen Hyperlipämie-Typen sind jedoch entsprechend dem jeweiligen Cholesterin/ Triglycerid-Quotienten Frequenzunterschiede von 18 bis $54 \mathrm{rel} \%$ vorhanden. Ihre auffällige Beziehung zum Körpergewicht, d. h. namentlich Übergewicht bei 
Personen mit bzw. ohne Hyperlipoproteinämie bedarf weiterer Abklärung.

Das Lp(a)-Lipoprotein findet sich entsprechend früheren Befunden in der Dichteklasse von $1,050-1,090 \mathrm{~g} / \mathrm{ml}$ $(27,6,29)$. Es ist durch eine leichte Dissoziation in vitro ausgezeichnet.

Der Hauptbestandteil des komplexen Lipoproteins konnte als LDL charakterisiert werden. Zusätzlich enthält es jedoch einen Proteinanteil, der Träger der Lp(a)-Antigendeterminante ist, sowie Albumin (21, 22). Aus Untersuchungen mit proteolytischen Enzymen (6) kann geschlossen werden, daß Albumin und $L_{p}(a)-$ Protein etwa 30\% des Proteinanteils bilden. Die restlichen $70 \%$ bestehen neben Apolipoprotein C (30) fast ausschließlich aus Apolipoprotein B. Lipidchemisch ist der Reichtum an Glykolipiden für das Lp(a)-Lipoprotein kennzeichnend. Abgesehen von Cerebrosiden (Abb. 9b) ist der Gehalt an Neuraminsäure (Hämatoside) - bezogen auf das Lipoproteineiweiß etwa $5 \mathrm{mal}$ größer als vergleichsweise in LDL (26). Das Verhältnis von Plasma- und Phosphorgehalt entspricht dem des Normalserums $(31,32)$. Bei den Neutrallipiden erscheint die hohe Konzentration an veresterten Polyensäuren (Cholesterinester) bemerkenswert.

Die biologische Bedeutung des charakterisierten komplexen Lipoproteins ist zur Zeit noch unbekannt. Ein Zusammenhang des Lp(a)-Lipoproteins mit dem HLASystem ist diskutiert worden (33), jedoch weist der offensichtliche Zusammenhang mit der Hyperlipoproteinämie eher auf eine Rolle des Lp(a)-Proteins im Lipidstoffwechsel hin. $\mathrm{Da}$ das $\mathrm{L}_{\mathrm{p}}$ (a)-Lipoprotein elektrophoretisch bevorzugt im Rückbildungsstadium einer Hyperlipoproteinämie nachweisbar wird - unabhängig ob spontan oder therapeutisch begünstigt - könnte insbesondere an einen Einfluß auf die Senkung der Serumlipide gedacht werden.

Umfangreiche Familienuntersuchungén $(20,34,35)$ haben gezeigt, daß die Konzentration des Lp(a)Lipoproteins unter genetischer Kontrolle steht. Zwischen individuellen Seren bestehen dabei erhebliche Schwankungen $(26,37,5)$; doch wurde bisher angenommen, daß die Konzentration im Serum eines individuellen Spenders relativ konstant ist (38).

Die vorliegende Untersuchung zeigt, daß die Konzentration des $L_{P}(a)$-Lipoproteins abhängig vom funktionellen Zustand des Organismus offensichtlich großen Schwankungen unterworfen sein kann. Dem Nachweis des Lp(a)-Lipoproteins kommt deshalb im Einvernehmen mit anderen Untersuchungsergebnissen $(37,5)$ vor allem quantitative Bedeutung zu. Er kann dazu beitragen, bestimmte Lipoproteinmuster differenzierter zu beurteilen.

Herrn T. KURODA möchten wir für seine technische Mitarbeit und der Deutschen Forschungsgemeinschaft für die finanzielle Unterstuitzung der Arbeit an dieser Stelle besonders danken.

\section{Literatur}

1. Rapp, W. \& KahlKe, W. (1968), Clin. Chim. Acta 19, 493-498. 2. Vogelberg, K. H. (1969), Klin. Wochenschr. 47, 722 . 3. Havel, R. J., Eder, H. A. \& Bragdon, J. H. (1955), J. Clin. Invest. 34, 1345-1353. - 4. UTERMANN, G. \& WIEGANDT, H. (1969), Humangenetik 8, 39-46. - 5. UTERMANN, G. \& WIEGANDT, H. (1970), Humangenetik 11, 66-71. - 6. UTERMANN, G. \& Wiegandi, H. (1971), Hoppe-Seylers Z. Physiol. Chem. 352, 938-946. - 7. Zlotkis, A., ZAK, W. \& BOYLE, A. J. (1953), J. Lab. Clin. Med. 41, 486-492. - 8. JAhnKe, K. \& Herberg, M. (1969), Ärztl. Lab. 15, 201-208. - 9. BarLETr, G. R. (1959), J. Biol. Chem. 234, 466-468. - 10. Leupold, F. H. \& BÜTTNER, H. (1954), Klin. Wochenschr. 32, 119-121. - 11. WINTERFELD, M. (1966), Dissertation, Köln. - 12. FolCH, J., LEES, M. \& Stanley, G. H. S. (1957), J. Biol. Chem. 226, 497-509. 13. Vogelberg, K. H. (1972), diese Z. 10, 172-173. - 14. Stoffel, W., Chu, F. \& Arrens, E. H. (1959), Analyt. Chem. 31, 307-310. - 15. Freeman, C. P. \& West, D. (1966), J. Lipid Res. 7, 324-327. - 16. vaN GeNT, C. M. (1968), Z. Analyt. Chem. 236, 344-350. - 17. Debuch, H., Mertens, W. \& Winterfeld, M. (1968), Hoppe-Seylers Z. Physiol. Chem. 349, 896-902. - 18. VANCE, D. E. \& Sweeley, C. C. (1967), J. Lipid. Res. 8, 621-630. - 19. VogelberG, K. H., UtermaNN, G. \& Gries, F. A. (1971), Symposion „Struktur, Stoffwechsel und klinische Aspekte der Lipoproteine des Blutes". Ulm, 1971. 20. Berg, K. (1963), Acta Path. Microbiol. Scand. 59, 369-382. 21. Erinholm, C., Simons, K. \& Garoff, H. (1972), in: Proteides of biological fluids 19, 191-196, edit. H. Peeters. - 22. UTERMANN, G., LIPP, K. \& WIEGANDT, H. (1972), Humangenetik 14, 142-150. - 23. Ellefson, R. D., Jiminez, B. J. \& SMITH, R. C. (1971), Mayo Clin. Proc. 46, 328-332. - 24. Papadopoulos, N. M. \& Kintzios, J. A. (1971), Clin. Chem. 17, 427-429. 25. Wille, L. E. \& Philirs, G. W. (1971), Clin. Genetics 2, 242-247. - 26. Simons, K., Ehnholm, C., Renkonen, O. \& Blotri, B. (1970), Acta Path. Microbiol. Scand. Section B 78 , 459-466. - 27. RIDER, A. K., LEVY, R. J. \& FREDRICKSON, D. S. (1970), Circulation 42, Suppl. III-10. - 28. BERG, K. (1966), Internationale $\mathrm{LP}_{\mathrm{p}}$-Arbeitstagung 28 . - 29. WIEGANDT, H., LIPP, K. \& Wendt, G. G. (1968), Hoppe-Seyler's Z. Physiol. Chem. 349, 489-494. - 30. GEISEN, H. P., SBIDEL, D. \& ROELCKE, D. (1971), Symposion über „Struktur, Stoffwechsel und klinische Aspekte der Lipoproteine des Blutes“. Ulm, 1971. - 31. THIELE, O. W. (1955), Z. Ges. Exp. Med. 125, 136-141. - 32. VogeLBerg, K. H. \& Debuch, H. (1971), Veth. Dtsch. Ges. Inn. Med. 77, 585-588. - 33. BERG, K. (1971), Clin. Genet. 2, 193-202. 34. RitrNer, C. \& WIRChMANN, D. (1967), Humangenetik 5 , 42-53. - 35. WENDT, G. G. (1967), Humangenetik 3, 269-272. 36. Ehnholm, C., Garofr, H., Stmons, K. \& Aro, A. (1971), Biochem. Biophys. Acta 236, 431-439. - 37. HaRvis, N. R. \& Schultz, J. S. (1970), Proc. Natl. Acad. Sci. 66, 99-103. 38. RIITNER, C. (1970), Habilitationsschrift, Bonn. - 39. SKIPSKI; V. P., BARday, M., Barday, R. K., Fetzer, V. A., Good, J. J. \& Archibald, F. M. (1967), Biochem. J. 104, 340-352.
Dr. K. H. Vogelberg 4000 Düsseldorf Moorenstr. 5 